\title{
The XENON project for dark matter direct detection at LNGS
}

\author{
Andrea Molinario ${ }^{1, a}$ on behalf of the XENON Collaboration \\ ${ }^{1}$ INFN-Laboratori Nazionali del Gran Sasso, via G. Acitelli 22, 67100 Assergi (AQ), Italy
}

\begin{abstract}
The XENON project at INFN Laboratori Nazionali del Gran Sasso, Italy, aims at dark matter direct detection with liquid xenon dual-phase time projection chambers. Latest results of XENON100 detector exclude various models of leptophilic dark matter. A search for low mass weakly interacting massive particles was also performed, lowering the energy threshold for detection to $0.7 \mathrm{keV}$ for nuclear recoils. The multi-ton XENON1T detector is fully installed and operating. It is expected to reach a sensitivity a factor 100 better than XENON100 with a 2 ton-year exposure.
\end{abstract}

\section{Introduction}

The XENON project at INFN Laboratori Nazionali del Gran Sasso (LNGS), Italy, aims at the direct detection of dark matter in the form of weakly interacting massive particles (WIMPs). In the XENON time projection chambers (TPCs), liquid xenon (LXe) is used both as a target and self-shielding medium for background reduction. The WIMP elastic scattering off Xe nuclei would produce low energy nuclear recoils at extremely low interaction rates.

The working principle of the XENON detectors is presented in figure 1. Photomultipler tubes (PMTs) arrays are placed on top and bottom of the TPC. Cathode, gate, and anode grids define electric fields in the TPC. When a particle interacts with Xe in the LXe volume, the interaction creates the excitation and ionization of Xe. Direct scintillation (S1) from the excitation and electron-ion recombination is detected by the PMTs immediately after the interaction. The electrons, escaping the recombination, drift upward due to the electric field. Those electrons are extracted into the gas volume by a high electric field between the gate and the anode, and then accelerated in gaseous xenon $(\mathrm{GXe})$. This creates Xe excitation in the gas volume. The scintillation signal in the gas phase (S2) is delayed relative to $\mathrm{S} 1$ by the drift time of the electrons through the liquide. The three-dimensional position of the event is reconstructed using the drift time and the S2 hit pattern on the top PMT array. This enables background suppression by selection of a fiducial volume. The ratio S2/S1 is different for electronic recoils (ERs), induced by electrons and $\gamma$, and nuclear recoils (NRs), induced by neutrons and dark matter particles. This feature allows to reject the most abundant electromagnetic backround and provides powerful background discrimination.

The XENON detectors have been operating at LNGS at an average depth of $3600 \mathrm{~m}$ water equivalent since 2006. The first operating detector has been XENON10 [1], with a total Xe mass of $25 \mathrm{~kg}$.

ae-mail: andrea.molinario@lngs.infn.it 
It was followed, since 2007, by XENON100 [2] with a total Xe mass of $161 \mathrm{~kg}$. This detector set a limit to the WIMP-nucleon spin-independent cross section of $\sigma_{S I}<1.1 \times 10^{-45} \mathrm{~cm}^{2}$ for a WIMP mass of $50 \mathrm{GeV} / \mathrm{c}^{2}[3]$.

The multi-ton scale phase of the project, XENON1T, with a total Xe mass of 3.2 tons, is fully installed and operating.

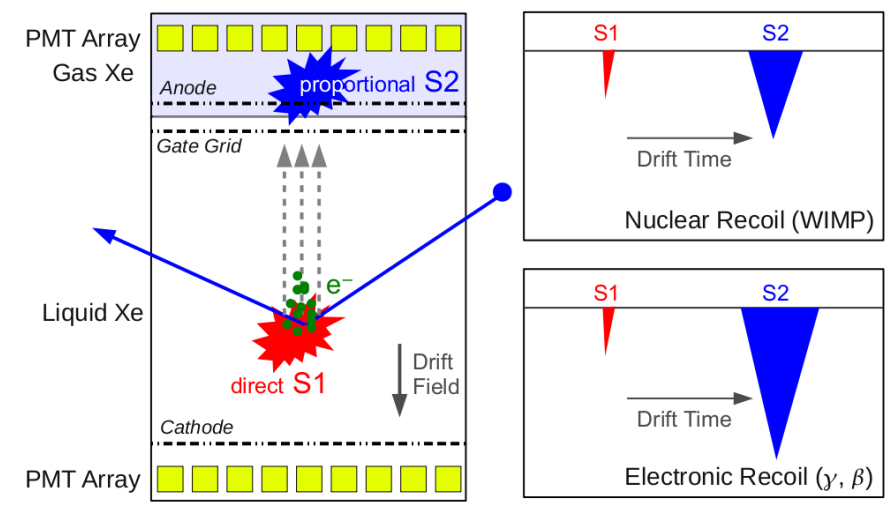

Figure 1. Left: working principle of the XENON liquid-gas dual-phase TPC. Right: sketch of the waveforms of nuclear recoils (WIMPs or neutrons) and electronic recoils ( $\gamma$ or $\beta$ background), showing the different ratio of the charge (S2) and light (S1) signals for the two types of events [2].

\section{Latest results from XENON100}

Latest results from XENON100 data analysis are presented in this section. All these analysis were performed on the 225 live-days data taking period with which the collaboration was able to set stringent limits on the spin-independent (SI) and spin-dependent (SD) WIMP-nucleon cross section [3] [4] [5], as well as for axion-induced electronic recoils [6].

\subsection{Exclusion of leptophilic dark matter models}

We tested the possibility that DAMA/LIBRA annual modulation [7] in the 2-6 keV energy range can be due to dark matter scattering on electrons, instead of scattering on nuclei [8]. In particular we contrast the DAMA/LIBRA signal, interpreted as WIMPs coupling to electrons through axialvector interactions [9] [10], with XENON100 data. For this analysis, the events belonging to the electronic recoil band in XENON100 and occurring in the $34 \mathrm{~kg}$ inner fiducial volume have been considered. Moreover, the study is performed using events from 70 summer live-days only, where the modulation effect is stronger. Figure 2 shows the comparison among the converted DAMA/LIBRA and the measured XENON100 energy spectra. A simple comparison of the integral counts in the energy interval from 2 to $14 \mathrm{PE}$, which corresponds to 2.0 to $5.9 \mathrm{keV}$ electronic recoil energy, excludes the DAMA/LIBRA signal as axial-vector coupling between WIMPs and electrons at $4.4 \sigma$ significance level, even considering all events from XENON100 background as signal candidates. XENON100 is also able to exclude DAMA/LIBRA signal as due to other two models. Luminous dark matter [11] is excluded at $4.6 \sigma$ and mirror dark matter [12] is excluded at $3.6 \sigma$. 


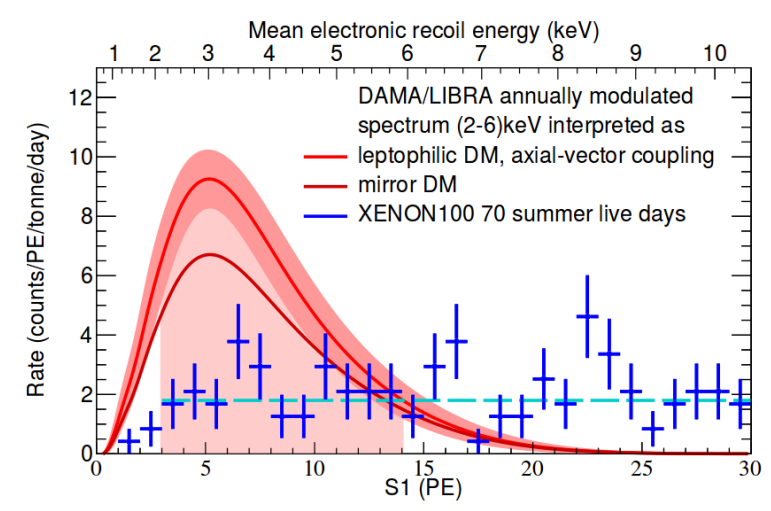

Figure 2. The DAMA/LIBRA modulated spectrum (red), interpreted as WIMPs scattering through axial-vector interactions, as it would be seen in the XENON100 detector. The $1 \sigma$ band includes statistical and systematic uncertainties. The DAMA/LIBRA modulated spectrum interpreted as luminous dark matter is very similar, whereas the interpretation as mirror dark matter is indicated separately (dark red). Blue points are XENON100 data from the 70 summer live days with their statistical uncertainty. The expected average XENON100 rate is also shown (dashed cyan). The shaded region from (3 to 14) PE was used to quantify the confidence level of exclusion [8].

\subsection{Search for event rate modulation}

XENON100 is the first dual-phase TPC operated stably for long-enough period to allow performing a study of annual modulation [13]. The study is performed again on the events belonging to the electronic recoil band in XENON100 and occurring in the $34 \mathrm{~kg}$ inner fiducial volume. The time modulation is tested at first with no constraints on the period for the single scatter events in the 2.0$5.8 \mathrm{keV}$ energy range. A rise in significance for a modulation at long periods is observed, but it is still below $3 \sigma$ local and $1 \sigma$ global (figure 3 left). This rise in significance is observed both in the single scatter and in the multiple scatters sample, in contrast with what expected if the modulation was due to dark matter scattering. If the period is fixed to 1 year, there is a $2.8 \sigma$ local significance. The phase of this modulation disfavors the dark matter interpretation at $2.5 \sigma$, and the DAMA/LIBRA signal is disfavored at $4.8 \sigma$, see figure 3 right.

\subsection{Search for low mass dark matter}

A dedicated analysis has been performed to search for low mass dark matter [14]. In order to lower the energy threshold, this analysis has been performed by using only the ionization signal S2. This choice prevents on one hand to discriminate the ER from the NR (through the S2/S1 ratio), on the other hand to get the vertical position of one event (using the time difference between S2 and S1). A NR equivalent energy scale has been reconstructed, with a charge yield based on the model in [15], with a cutoff below $0.7 \mathrm{keV}$. Without a background model, we interprete all events surviving quality cuts as potential dark matter candidates. At a WIMP mass of $6 \mathrm{GeV} / \mathrm{c}^{2}$, SI WIMP-nucleon interaction cross sections of $1.4 \cdot 10^{-41} \mathrm{~cm}^{2}$ are excluded, figure 4 . 

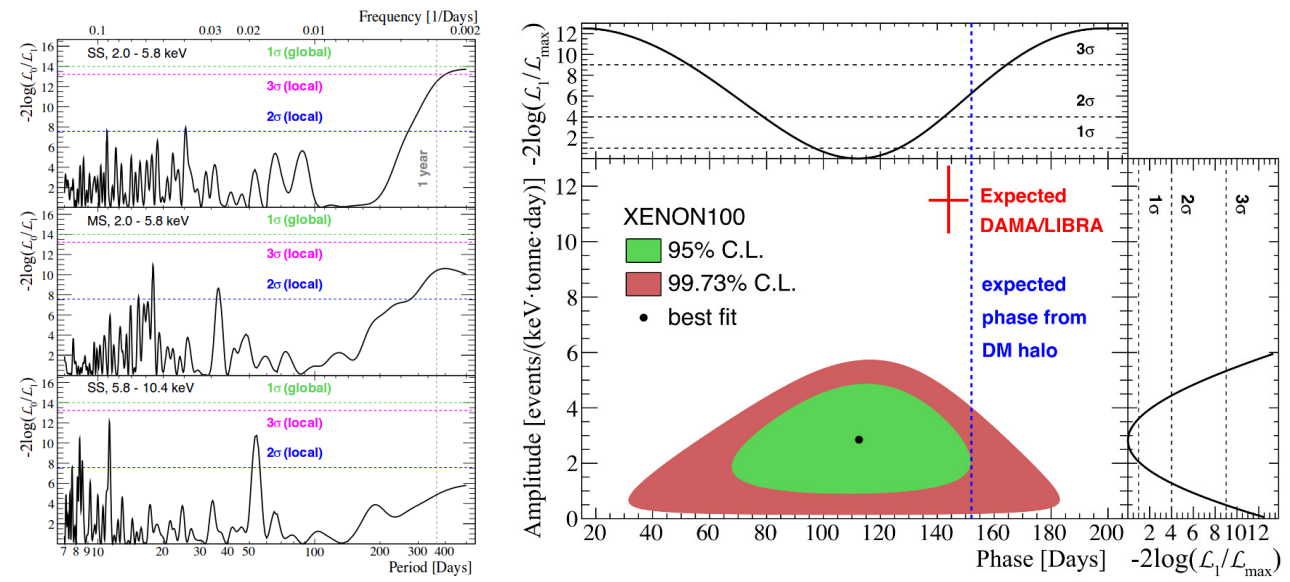

Figure 3. Left: The significance as a function of modulation period for single scatters (SS) in the low-E region (top panel), multiple scatters (MS) in the low-E region (middle panel) and single scatters (SS) in the higher energy region (bottom panel). The phase is unconstrained. Right: The XENON100 best-fit and 95\% and 99.73\% confidence level contours as a function of amplitude and phase relative to January 1,2011 , for period $\mathrm{P}=1$ year. The expected DAMA/LIBRA signal with statistical uncertainties only and the phase expected from a standard dark matter halo are overlaid for comparison. Top and side panels show significance as a function of phase and amplitude, respectively [13].

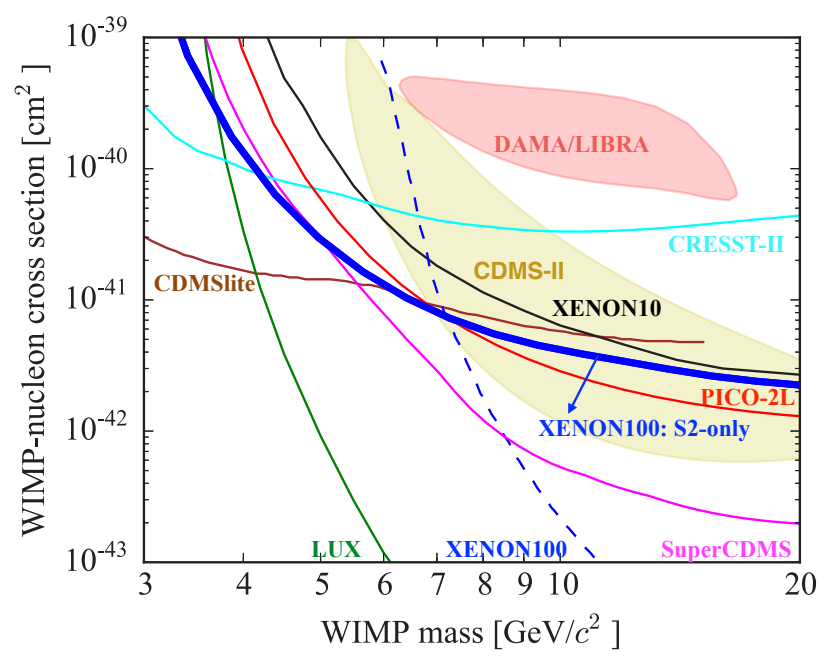

Figure 4. WIMP exclusion limit on the SI WIMP-nucleon scattering cross section at 90\% C.L. The limit from the search for low mass dark matter is shown with the thick blue line [14]. 


\section{XENON1T detector and expected sensitivity}

XENON1T is the current phase of the XENON program. With a total Xe mass of 3.2 tons, XENON1T is expected to reach a sensitivity about a factor 100 higher than XENON100 in 2 ton.year exposure.

The TPC of XENON1T is $96 \mathrm{~cm}$ height and $96 \mathrm{~cm}$ diameter, filled with $\sim 2$ tons of liquid xenon (figure 5, middle). A total of 248 PMTs are distributed at the top and bottom of the TPC. The detector is hosted in a $10 \mathrm{~m}$ height and $10 \mathrm{~m}$ diameter water tank acting as a passive shield against natural radioactivity. Additionally, the water tank is operating as an active muon veto, being instrumented with 84 PMTs to detect both passing cosmic muons and muon-induced cascades by Cherenkov effect [16].
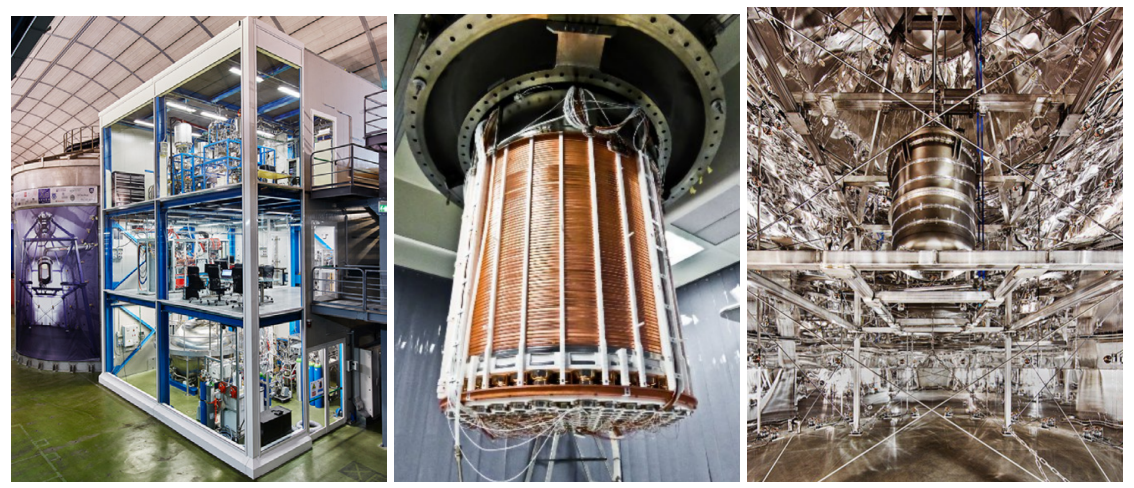

Figure 5. Left: a global view of the XENON1T infrastructure at LNGS. Middle: the TPC of XENON1T. Right: the cryostat hanging in the center of the water tank.
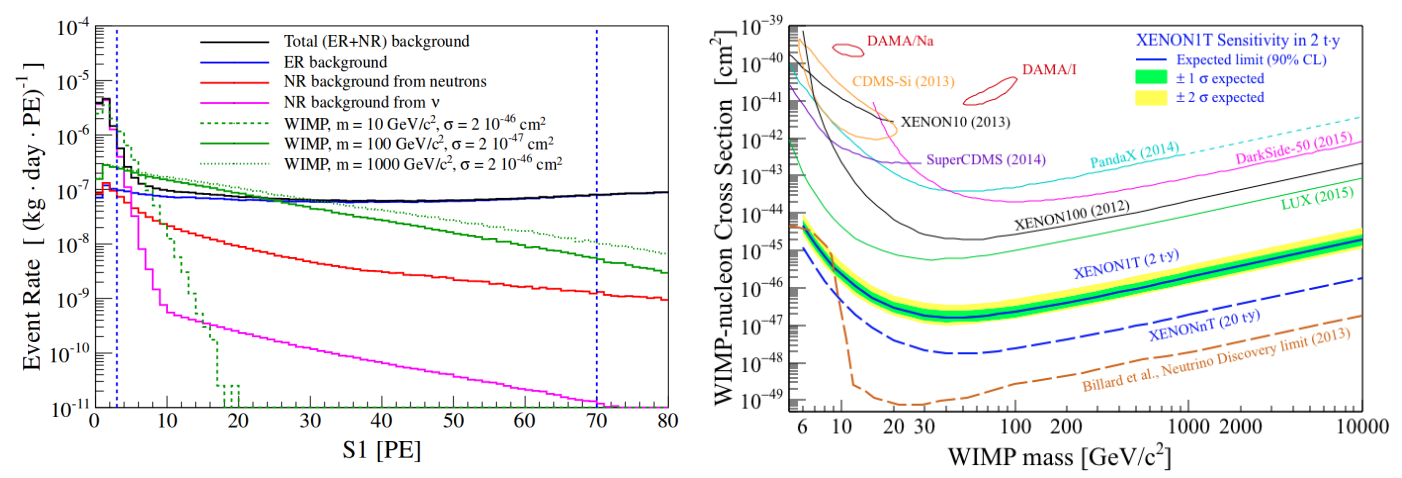

Figure 6. Left: Simulated S1 spectra of background compared with the expectations from WIMP of different masses. These are obtained considering single scatter in $1 \mathrm{t}$ fiducial volume, energy range [1-12] $\mathrm{keV}_{e e}$ [4-50] $\mathrm{keV}_{n}, 99.75 \%$ ER discrimination, $40 \%$ NR acceptance. Right: XENON1T sensitivity to SI WIMP-nucleon interaction with a 2 ton·year exposure [17].

A dedicated Geant4-based XENON1T Monte Carlo simulation has been developed, including radioactivities of all detector materials, and energy deposition into S1 and S2 [17]. Figure 6 shows 
the expected S1 spectra of backgrounds and WIMP signals as a function of S1 (left), and the expected sensitivity to SI WIMP-nucleon interaction (right). The expected XENON1T 90\% C.L. SI WIMPnucleon cross section upper limit reaches better than $2 \times 10^{-47} \mathrm{~cm}^{2}$ at WIMP mass of $50 \mathrm{GeV} / \mathrm{c}^{2}$ with 2 ton·year exposure.

\section{Summary}

The XENON project aims at dark matter direct detection with liquid xenon dual-phase TPCs. Latest analysis of XENON100 data excluded various models of leptophilic dark matter. A search for low mass WIMPs was also performed, looking at ionization signals (S2) in order to lower the energy threshold to $0.7 \mathrm{keV}_{n r}$. The XENON1T detector is fully installed and operating at LNGS. It is expected to reach a sensitivity a factor 100 better than XENON100 with 2 ton-year exposure.

\section{References}

[1] J. Angle et al. (XENON Collaboration), Phys. Rev. Lett. 100021303 (2008)

[2] E. Aprile et al. (XENON Collaboration), Astropart. Phys. 35, 573-590 (2012)

[3] E. Aprile et al. (XENON Collaboration), Submitted to PRD. arXiv:1609.06154

[4] E. Aprile et al. (XENON Collaboration), Phys. Rev. Lett. 109, 181301 (2012)

[5] E. Aprile et al. (XENON Collaboration), Phys. Rev. Lett. 111, 021301 (2013)

[6] E. Aprile et al. (XENON Collaboration), Phys. Rev. D Part. Fields Gravit. Cosmol. 90, 062009 (2014)

[7] R. Bernabei et al., Eur. Phys. J. C 73, 2648 (2013)

[8] E. Aprile et al. (XENON Collaboration), Science 2015 vol. 349 no. 6250 pp. 851-854

[9] J. Kopp, V. Niro, T. Schwetz, J. Zupan, Phys. Rev. D Part. Fields Gravit. Cosmol. 80, 083502 (2009)

[10] N. F. Bell, Y. Cai, R. K. Leane, A. D. Medina, Phys. Rev. D Part. Fields Gravit. Cosmol. 90, 035027 (2014)

[11] B. Feldstein, P. W. Graham, S. Rajendran, Phys. Rev. D Part. Fields Gravit. Cosmol. 82, 075019 (2010)

[12] R. Foot, Int. J. Mod. Phys. A 29, 1430013 (2014)

[13] E. Aprile et al. (XENON Collaboration), Phys. Rev. Lett. 115, 091302 (2015)

[14] E. Aprile et al. (XENON Collaboration), Phys. Rev. D 94, 092001 (2016)

[15] F. Bezrukov, F. Kahlhoefer, M. Lindner, Astropart. Phys. 35, 119-127 (2011)

[16] E. Aprile et al. (XENON Collaboration), JINST 9, P11006 (2014)

[17] E. Aprile et al. (XENON Collaboration), JCAP 04 (2016) 027 